\title{
Prevalence and distribution of ringed sideroblasts in primary myelodysplastic syndromes
}

\author{
SK JUNEJA, M IMBERT, F SIGAUX, H JOUAULT, C SULTAN \\ From the Service Central d'Hématologie-Immunologie - Hôpital Henri Mondor 94010 Creteil, France
}

SUMMARY In order to determine the prevalence and percentage distribution of ringed sideroblasts in primary myelodysplastic syndromes, the results of Prussian blue staining were analysed in 133 cases. Ringed sideroblasts ranging from 1 to $86 \%$ of cells were found in $76(57 \%)$ cases. The cases of primary myelodysplastic syndrome corresponding to the group entitled "acquired idiopathic sideroblastic anaemia" had between 21 and $86 \%$ ringed sideroblasts; these were also found in $40 \%(26 / 65)$ cases corresponding to refractory anaemia with excess of blasts. Seven of the 22 cases having morphological features of refractory anaemia with excess of blasts in transformation had ringed sideroblasts. It would appear that cases of acquired idiopathic sideroblastic anaemia have at least $20 \%$ ringed sideroblasts; they also seem to occur frequently in refractory anaemia with excess of blasts.

Myelodysplastic syndromes are considered as myeloid stem cell disorders characterised by qualitative and quantitative abnormalities of erythrocytes, granulocytes, platelets and their precursors. They usually present peripheral blood anaemia, leucopenia and thrombocytopenia alone or in various combinations with a normo- or hypercellular marrow. The percentage distribution of ringed sideroblasts in acquired idiopathic sideroblastic anaemia and their prevalence in other myelodysplastic syndromes is not well documented. Further the occurrence of a large number of ringed sideroblasts in myelodysplastic syndromes other than in acquired idiopathic sideroblastic anaemia may lead to diagnostic problems. This study was therefore carried out to determine the relative number of ringed sideroblasts in various primary myelodysplastic syndromes.

\section{Material and methods}

One hundred and thirty-three cases of primary myelodysplastic syndrome defined according to criteria previously described, ${ }^{1}$ were studied. Thirteen cases of myelodysplastic syndrome with a history of chemotherapy or radiotherapy, or both, were not included in the study; five cases in which Prussian blue staining had not been done were also omitted. The ringed sideroblasts were defined as erythroblasts containing five or more Prussian blue positive granules covering one third or more of the circumference of the nucleus. The percentage of ringed sideroblasts was calculated from 100 erythroblasts. The percentage of various bone marrow cells was calculated from the 300 nucleated cells counted. The term blast was applied to myeloblasts with or without granules and to blasts of various sizes which may be unclassifiable. Recognisable promonocytes, proerythroblasts and megakaryoblasts were not included in the above category. The cases were divided into three groups:

Group 1 Those cases with $<5 \%$ blasts in the marrow and $<1 \%$ circulating blasts.

Group 2 Those cases with 5-20\% blasts in the marrow and $<5 \%$ circulating blasts.

Group 3 Those cases with $20-30 \%$ blasts in the marrow or $5-30 \%$ marrow blasts but with $\geqslant 5 \%$ circulating blasts or with Auer rods.

Routine haematological examinations were performed according to standard methods. ${ }^{23}$ Marked dyserythropoiesis was defined as the presence of two or more of the following morphological abnormalities in the majority of the erythroblasts: gigantism, multinuclearity, asynchrony of maturation between the nucleus and cytoplasm, cytoplasmic vacuolation, karyorrhexis, nuclear budding, basophilic stippling, Howell-Jolly bodies and megaloblastic changes.

Marked dysgranulopoiesis was defined by the presence of prominent degranulation in the majority of the granulocytes or the presence of two or more of the following morphologic abnormalities in the 


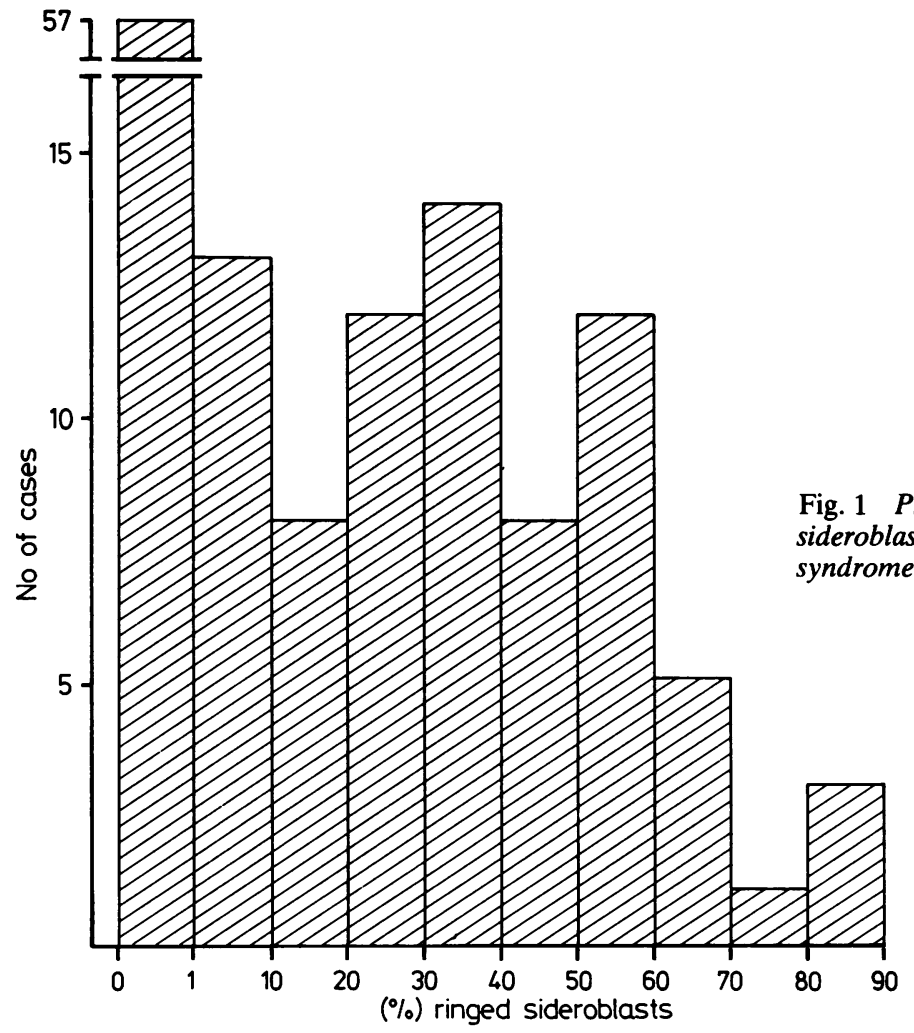

majority of the granulocytes: degranulation, abnormal granules, pseudo-Pelger anomaly, abnormal promyelocytes and dystrophic blasts. Dysthrombopoiesis was considered to be marked when the majority of the megakaryocytes were morphologically abnormal-that is micromegakaryocytes, megakaryocytes with fragmented nucleus, dystrophic megakaryocytes. Statistical analysis was performed using the $\chi^{2}$ test, analysis of variance and Student's $t$ test.

\section{Results}

The prevalence and percentage distribution of ringed sideroblasts in 133 cases of primary myelodysplastic syndrome is shown in Figure 1.

Seventy-six cases $(57 \%)$ had ringed sideroblasts in the marrow. The percentage of ringed sideroblasts varied from $1-86 \%$.

Five morphological parameters (i) ringed sideroblasts $>20 \%$; (ii) erythroid hyperplasia ( $\geqslant 30 \%$ erythroblasts); (iii) marked dyserythropoiesis; (iv) marked dysgranulopoiesis; (v) marked dysthrombopoiesis, were compared among the three groups (Table). The percentage distribution of ringed sideroblasts in the three groups is shown in Fig. 2. Forty-three $(93 \%)$ of the 46 cases in group 1 had ringed sideroblasts, varying from 7-86\%. In group $2,26(40 \%)$ had ringed sideroblasts varying from $1-62 \%$. Only seven of the 22 cases in group 3 had ringed sideroblasts varying from 1-78\%. Compared to groups 2 and 3 , significantly more cases of group

Morphological abnormalities in primary myelodysplastic syndrome

\begin{tabular}{llllll}
\hline Groups & No of cases & $\begin{array}{l}\text { Marked } \\
\text { dyserythropoiesis }\end{array}$ & $\begin{array}{l}\text { Marked } \\
\text { dysgranulopoiesis }\end{array}$ & $\begin{array}{l}\text { Marked } \\
\text { dysthrombopoiesis }\end{array}$ & $\begin{array}{l}\text { Cases having } \\
\text { > 20\% ringed sideroblasts } \geqslant 30 \% \text { erythroblasts }\end{array}$ \\
\hline 1 & 46 & $38(83)$ & $3(6)$ & $2(4)$ & $40(87)$ \\
2 & 65 & $20(31)$ & $48(74)$ & $28(43)$ & $13(20)$ \\
3 & 22 & $2(9)$ & $17(77)$ & $12(54)$ & $17(93)$ \\
$26)$ & $5(23)$ & Cases having & $(9)$ \\
\hline
\end{tabular}

Percentages are shown in parentheses. 


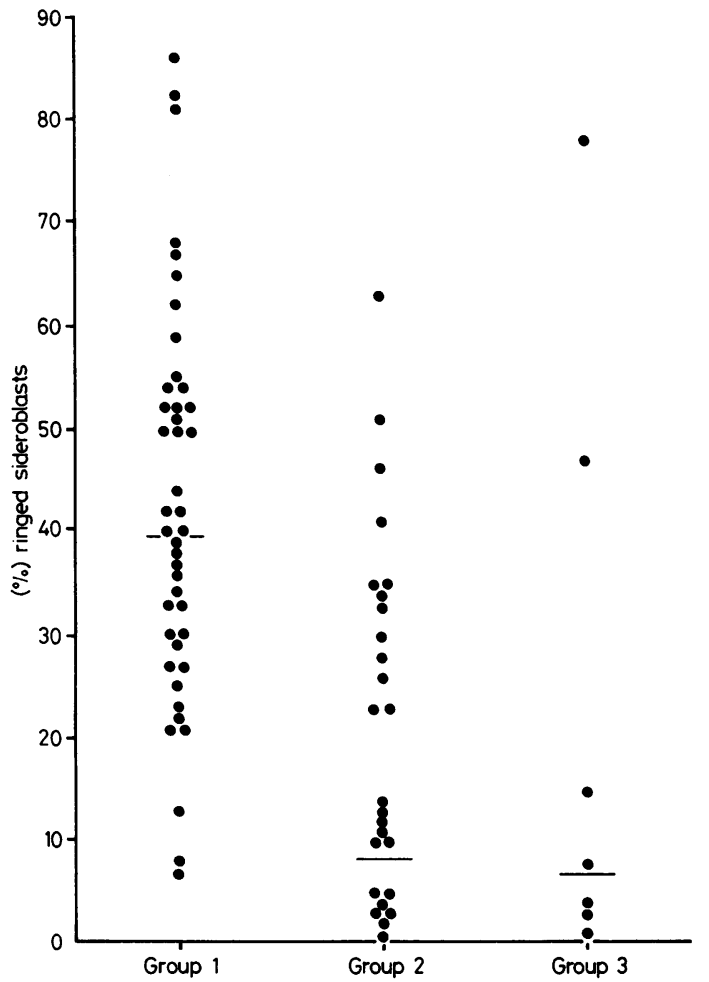

Fig. 2 Distribution of ringed sideroblasts in the three groups of primary myelodysplastic syndrome.

1 had (a) ringed sideroblasts greater than $20 \%$ (p $<$ 0.001 ) (b) erythroid hyperplasia ( $<<0.001)$ (c) marked dyserythropoiesis $(\mathrm{p}<0.001)$. On the other hand, marked dysgranulopoiesis and marked dysthrombopoiesis were present in many more cases of groups 2 and 3 as compared to group $1(p<0.001)$.

No significant difference was, however, noted between the group 2 and group 3, in the above morphological parameters except marked dyserythropoiesis $(\mathrm{p}<0.05)$.

\section{Discussion}

Ringed sideroblasts are erythroblasts containing Prussian blue positive ferruginous granules arranged in a ring around the nucleus. This characteristic distribution is because of iron in the mitochondria which are present in a perinuclear position. ${ }^{4}$ The ineffective erythropoiesis observed in the primary myelodysplastic syndrome especially in acquired idiopathic sideroblastic anaemia, ${ }^{5}$ is due, at least partly, to these iron laden mitochondria which are not only morphologically but also functionally abnormal, thus resulting in cell death. ${ }^{6}$ Ringed sideroblasts are distinctly abnormal and are not found in a normal marrow. ${ }^{6}$ A variety of haematological and non-haematological conditions may be associated with the ringed sideroblasts in the marrow.?

The prevalence and percentage distribution of ringed sideroblasts in primary myelodysplastic syndrome have not been well documented except in acquired idiopathic sideroblastic anaemia where they form essential diagnostic criteria. They were found in a little more than half the cases in the present study.

A great majority of the cases with less than $5 \%$ marrow blasts had more than $20 \%$ ringed sideroblasts. These 40 cases would correspond to the group described as acquired idiopathic sideroblastic anaemia in published reports.$^{8-10} \mathrm{~A}$ variable percentage of ringed sideroblasts in acquired idiopathic 8 sideroblastic anaemia has been reported. ${ }^{810}$ In the 음 present study, ringed sideroblasts varied from 21$86 \%$ and $45 \%(18 / 40)$ had $>50 \%$ ringed sideroblasts. The remaining six cases of group 1, of which three lacked ringed sideroblasts and the remaining $\vec{\bullet}$ three had between 7 and $13 \%$ ringed sideroblasts, would correspond to the group described as refractory cytopenia; the three latter cases having ringed sideroblasts, had dysgranulopoiesis and dysthrombopoiesis.

In the present study, group 2 would correspond to that described as refractory anaemia with excess of blasts. ${ }^{8}$ An important finding is the presence of ringed sideroblasts in $40 \%(26 / 65)$ and even $>20 \%$ in one-fifth (13/65) of such cases. This finding is in marked contrast to that reported recently. ${ }^{5}$ In the latter study, none of the cases of refractory anaemia with excess of blasts had $>5 \%$ ringed sideroblasts. This difference may be in part due to the slightly different criteria used for diagnosing this type of anaemia. Cases of refractory anaemia with excess of blasts with $>20 \%$ ringed sideroblasts can be differentiated from acquired idiopathic sideroblastic anaemia by the higher blast count and the presence of usually marked dysgranulopoiesis and dysthrombopoiesis in the former. This differentiation is $N$ necessary because of worse prognosis for the cases of refractory anaemia with excess of blasts. ${ }^{11}$

The 22 cases in group 3 correspond to the refractory anaemia with excess of blasts in transformation. ${ }^{8}$ Though seven of these cases had ringed sideroblasts, more cases need to be studied for better appreciation of their incidence and prevalence in this group.

From the data presented, it appears that among the primary myelodysplastic syndromes, the occurrence of ringed sideroblasts is not limited to acquired idiopathic sideroblastic anaemia and they occur also 
in other myelodysplastic syndromes. In our experience, the cases of acquired idiopathic sideroblastic anaemia have at least $20 \%$ ringed sideroblasts and a higher percentage is observed in the majority of them. Ringed sideroblasts also seem to occur frequently in refractory anaemia with excess of blasts.

\section{References}

${ }^{1}$ Sultan C. Dysmyelopoietic syndromes. In: Gralnick HR (moderator). Classification of acute leukaemia. Ann Intern Med 1977;87:740-53.

2 Dacie JV, Lewis SM. Practical haematology. 5th ed. Edinburgh: Churchill Livingstone, 1975.

${ }^{3}$ Sultan C, Priolet G, Beuzard Y, Rosa R, Josso F. Techniques en Hématologie. 2nd ed. Paris: Flammarion Médecine Sciences, 1978.

4 Bessis MC, Jensen WN. Sideroblastic anaemia, mitochondria and erythroblastic iron. Br J Haematol 1965;11:49-51.

${ }^{5}$ Cazzola M, Barosi G, Berzuini C, et al. Quantitative evaluation of erythropoietic activity in dysmyelopoietic syndromes. $\mathrm{Br} \mathrm{J}$ Haematol 1982;50:55-62.

- Cartwright GE, Deiss A. Sideroblasts, siderocytes and sideroblastic anaemia. $N$ Engl J Med 1975;292:185-93.

7 Sigaux F, Ricard MF, Imbert M, Sultan C. Les anémies sidéroblastiques acquises idiopathiques. Pathol Biol (Paris) 1979;27:421-32.

${ }^{8}$ Bennett JM, Catovsky D, Daniel MT. Proposals for the classification of the myelodysplastic syndromes. Br J Haematol 1982;51:189-99.

' Bottomley SS. Sideroblastic anaemia. Clin Haematol 1982; 11:389-409.

${ }^{10}$ Cheng DS, Kushner JP, Wintrobe MM. Idiopathic refractory sideroblastic anemia: Incidence and risk factors for leukemic transformation. Cancer 1979;44:724-31.

"Coiffier B, Adeleine P, Viala JJ, et al. Dysmyelopoietic syndromes: a search for prognostic factors in 193 patients. Cancer (in press).

Requests for reprints to: Professor C Sultan, Service Central d'Hematologie-Immunologie, Hôpital Henri Mondor 94010 Creteil, France. 